\title{
Aspidofractinine and Eburnane Alkaloids from a North \\ Borneo Kopsia. Ring-Contracted, Additional Ring-Fused, and Paucidactine-type Aspidofractinine Alkaloids from $K$. pauciflora
}

Wai-Sum Yap, ${ }^{\dagger}$ Chew-Yan Gan, ${ }^{\ddagger}$ Kae-Shin Sim, ${ }^{\S}$ Siew-Huah Lim, ${ }^{\dagger}$ Yun-Yee Low, $^{\dagger}$ and TohSeok $\mathrm{Kam}^{\dagger *}$

${ }^{\dagger}$ Department of Chemistry and ${ }^{\S}$ Institute of Biological Sciences, University of Malaya, 50603

Kuala Lumpur, Malaysia

${ }^{\ddagger}$ University Malaysia of Computer Science and Engineering, Jalan Alamanda 2, Precint 1, 62000

Putrajaya, Malaysia 


\section{List of Supporting Information}

Figure S1 $\quad{ }^{1} \mathrm{H}$-NMR spectrum of paucidirinine (1) $\left(\mathrm{CDCl}_{3}, 400 \mathrm{MHz}\right)$

Figure S2 $\quad{ }^{13} \mathrm{C}-\mathrm{NMR}$ spectrum of paucidirinine (1) $\left(\mathrm{CDCl}_{3}, 100 \mathrm{MHz}\right)$

Figure S3 $\quad{ }^{1} \mathrm{H}$-NMR spectrum of paucidirisine (2) $\left(\mathrm{CDCl}_{3}, 400 \mathrm{MHz}\right)$

Figure S4 ${ }^{13} \mathrm{C}-\mathrm{NMR}$ spectrum of paucidirisine (2) $\left(\mathrm{CDCl}_{3}, 100 \mathrm{MHz}\right) \quad 6$

Figure S5 $\quad{ }^{1} \mathrm{H}-\mathrm{NMR}$ spectrum of paucidactinine (3) $\left(\mathrm{CDCl}_{3}, 400 \mathrm{MHz}\right) \quad 7$

Figure S6 $\quad{ }^{13} \mathrm{C}$-NMR spectrum of paucidactinine (3) $\left(\mathrm{CDCl}_{3}, 100 \mathrm{MHz}\right)$

Figure S7 $\quad{ }^{1} \mathrm{H}$-NMR spectrum of pauciduridine (4) $\left(\mathrm{CDCl}_{3}, 400 \mathrm{MHz}\right) \quad 9$

Figure S8 $\quad{ }^{13} \mathrm{C}$-NMR spectrum of pauciduridine (4) $\left(\mathrm{CDCl}_{3}, 100 \mathrm{MHz}\right) \quad 10$

Figure S9 $\quad{ }^{1} \mathrm{H}-\mathrm{NMR}$ spectrum of paucidactine D (5) $\left(\mathrm{CDCl}_{3}, 400 \mathrm{MHz}\right) \quad 11$

Figure S10 ${ }^{13} \mathrm{C}$-NMR spectrum of paucidactine D (5) $\left(\mathrm{CDCl}_{3}, 100 \mathrm{MHz}\right)$

Figure S11 ${ }^{1} \mathrm{H}$-NMR spectrum of paucidactine E (6) $\left(\mathrm{CDCl}_{3}, 400 \mathrm{MHz}\right)$

Figure S12 ${ }^{13} \mathrm{C}$-NMR spectrum of paucidactine E (6) $\left(\mathrm{CDCl}_{3}, 100 \mathrm{MHz}\right) \quad 14$

Figure S13 $\quad{ }^{1} \mathrm{H}$-NMR spectrum of paucidisine (7) $\left(\mathrm{CDCl}_{3}, 400 \mathrm{MHz}\right) \quad 15$

Figure S14 ${ }^{13} \mathrm{C}$-NMR spectrum of paucidisine (7) $\left(\mathrm{CDCl}_{3}, 100 \mathrm{MHz}\right) \quad 16$

Figure S15 $\quad{ }^{1} \mathrm{H}-\mathrm{NMR}$ spectrum of (-)-19-oxoisoeburnamine (8) $\left(\mathrm{CDCl}_{3}, 400 \mathrm{MHz}\right) \quad 17$

Figure S16 $\quad{ }^{13}$ C-NMR spectrum of (-)-19-oxoisoeburnamine (8) $\left(\mathrm{CDCl}_{3}, 100 \mathrm{MHz}\right) \quad 18$

Figure S17 $\quad{ }^{1} \mathrm{H}$-NMR spectrum of (-)-19(R)-hydroxyeburnamenine (9) $\left(\mathrm{CDCl}_{3}, 400 \mathrm{MHz}\right) \quad 19$

Figure S18 $\quad{ }^{13} \mathrm{C}$-NMR spectrum of (-)-19(R)-hydroxyeburnamenine (9) $\left(\mathrm{CDCl}_{3}, 100 \mathrm{MHz}\right) \quad 20$

Figure S19 $\quad{ }^{1} \mathrm{H}$-NMR spectrum of (-)-19(R)-hydroxy-O-ethylisoeburnamine (10) $\left(\mathrm{CDCl}_{3}, \quad, \quad 21\right.$ $400 \mathrm{MHz})$

Figure S20 $\quad{ }^{13} \mathrm{C}$-NMR spectrum of (-)-19(R)-hydroxy-O-ethylisoeburnamine (10) $\left(\mathrm{CDCl}_{3}, \quad 22\right.$ $100 \mathrm{MHz})$ 


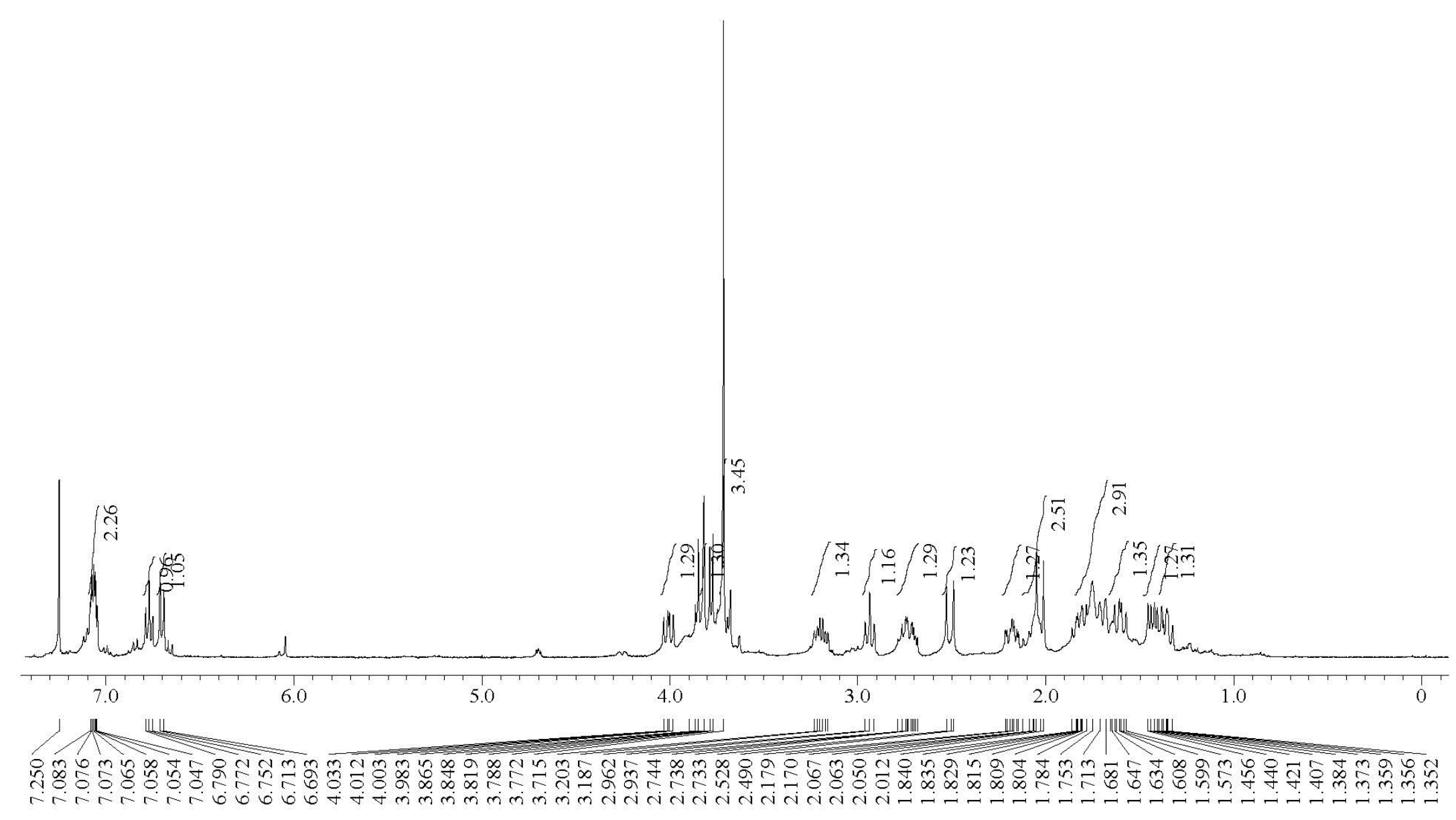

Figure S1. ${ }^{1} \mathrm{H}$ NMR Spectrum of Paucidirinine (1) $\left(\mathrm{CDCl}_{3}, 400 \mathrm{MHz}\right)$ 


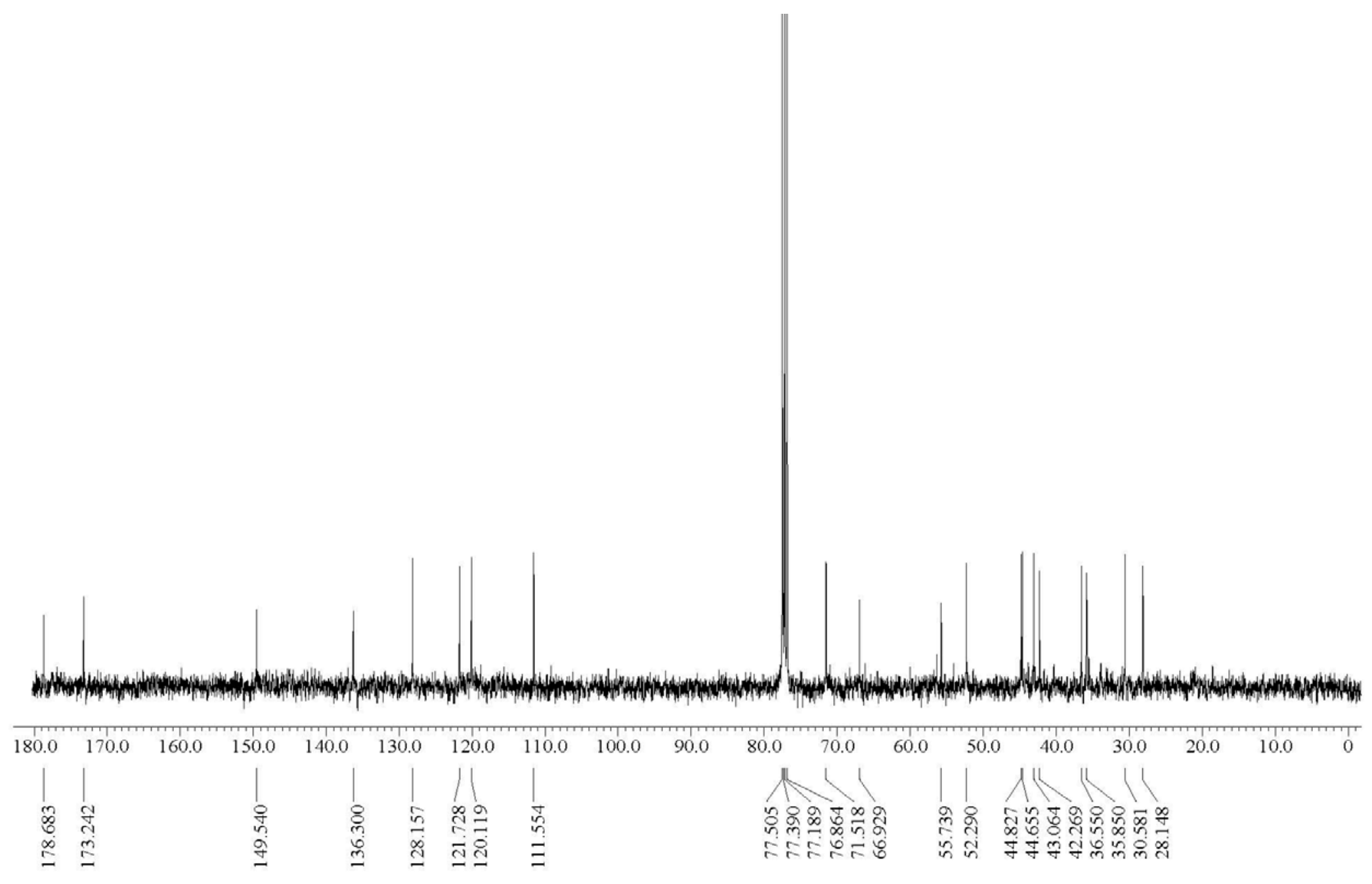

Figure S2. ${ }^{13} \mathrm{C}$ NMR Spectrum of Paucidirinine (1) $\left(\mathrm{CDCl}_{3}, 100 \mathrm{MHz}\right)$ 


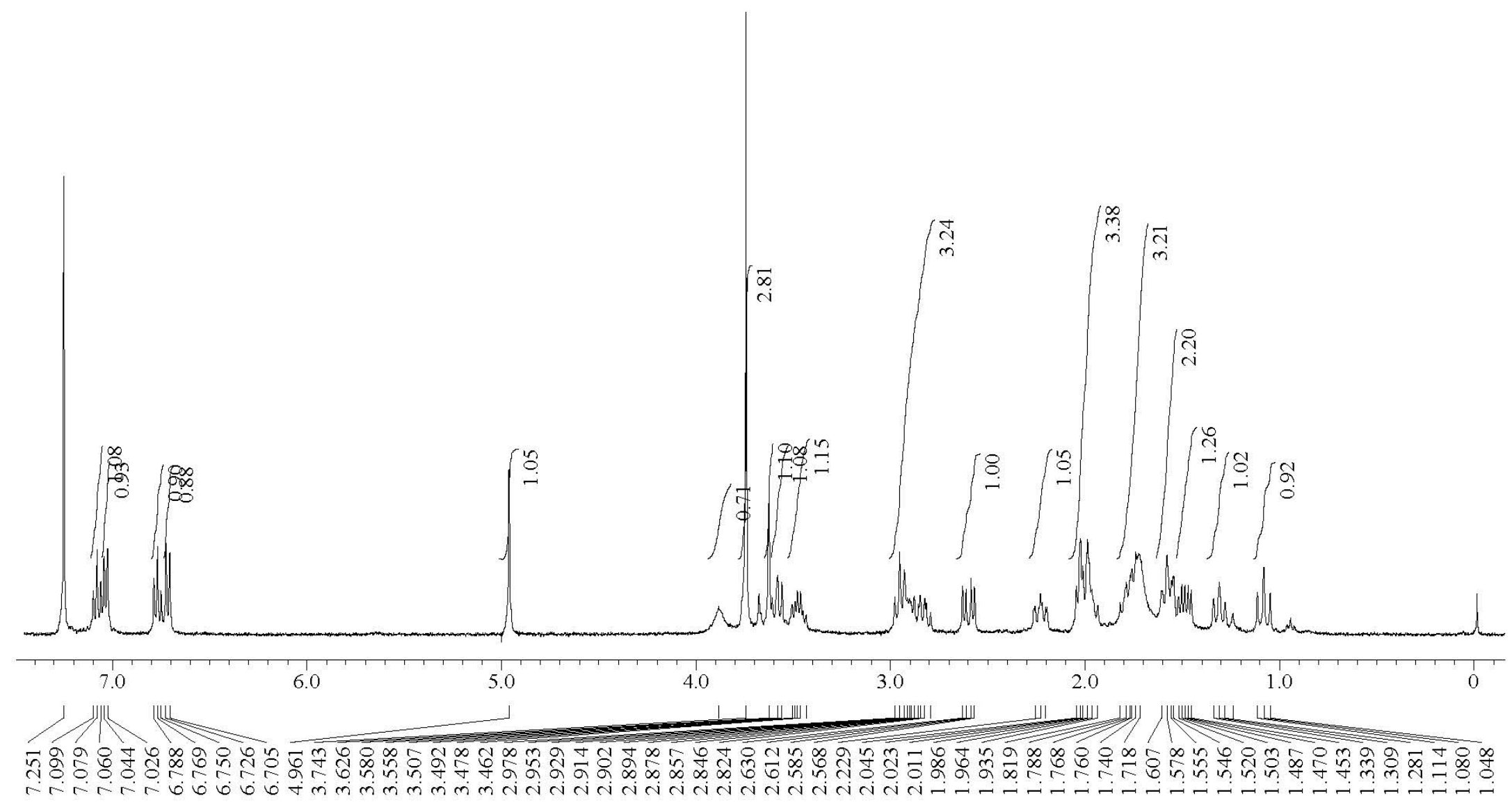

Figure S3. ${ }^{1} \mathrm{H}$ NMR Spectrum of Paucidirisine (2) $\left(\mathrm{CDCl}_{3}, 400 \mathrm{MHz}\right)$ 


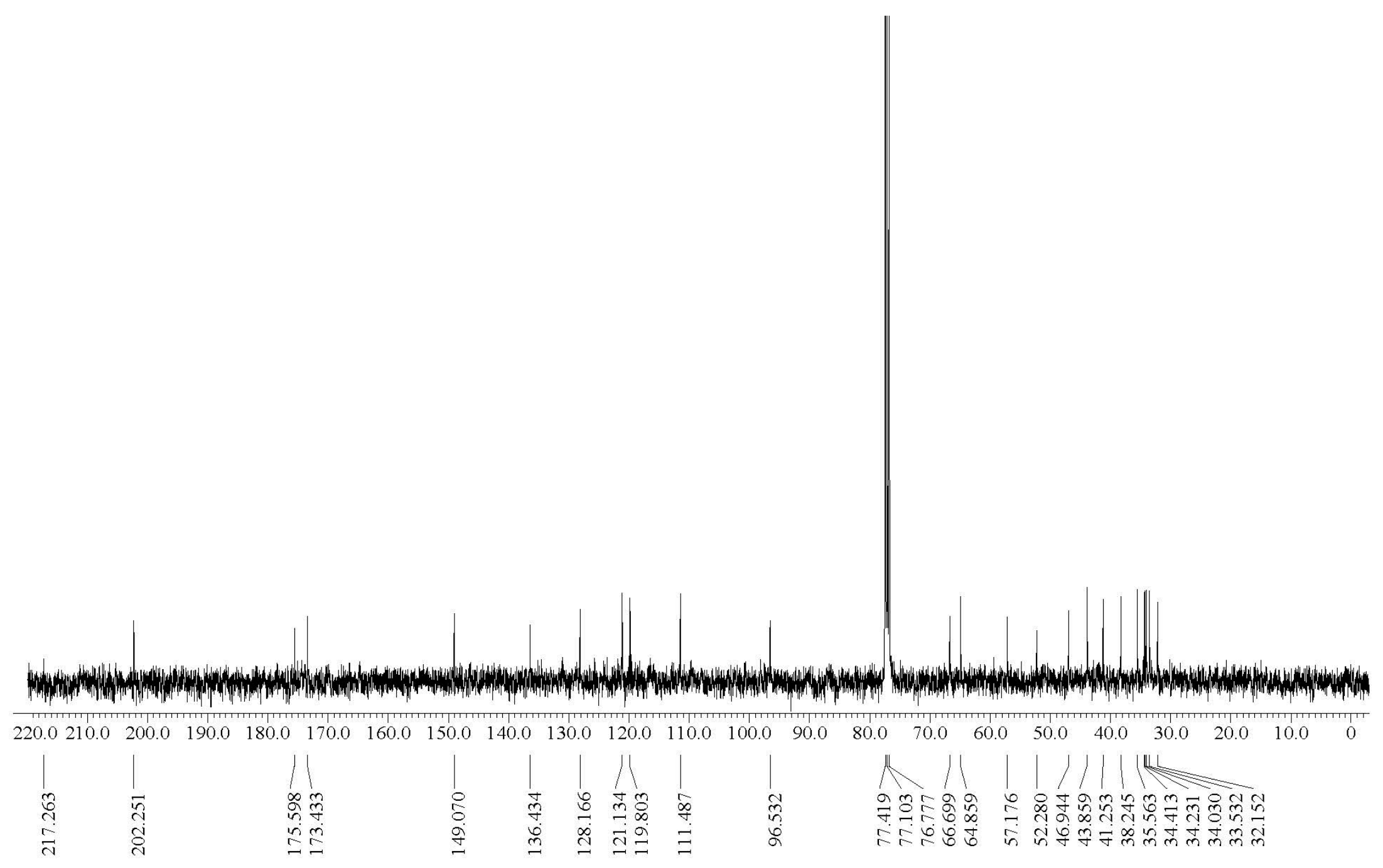

Figure S4. ${ }^{13} \mathrm{C}$ NMR Spectrum of Paucidirisine (2) $\left(\mathrm{CDCl}_{3}, 100 \mathrm{MHz}\right)$ 


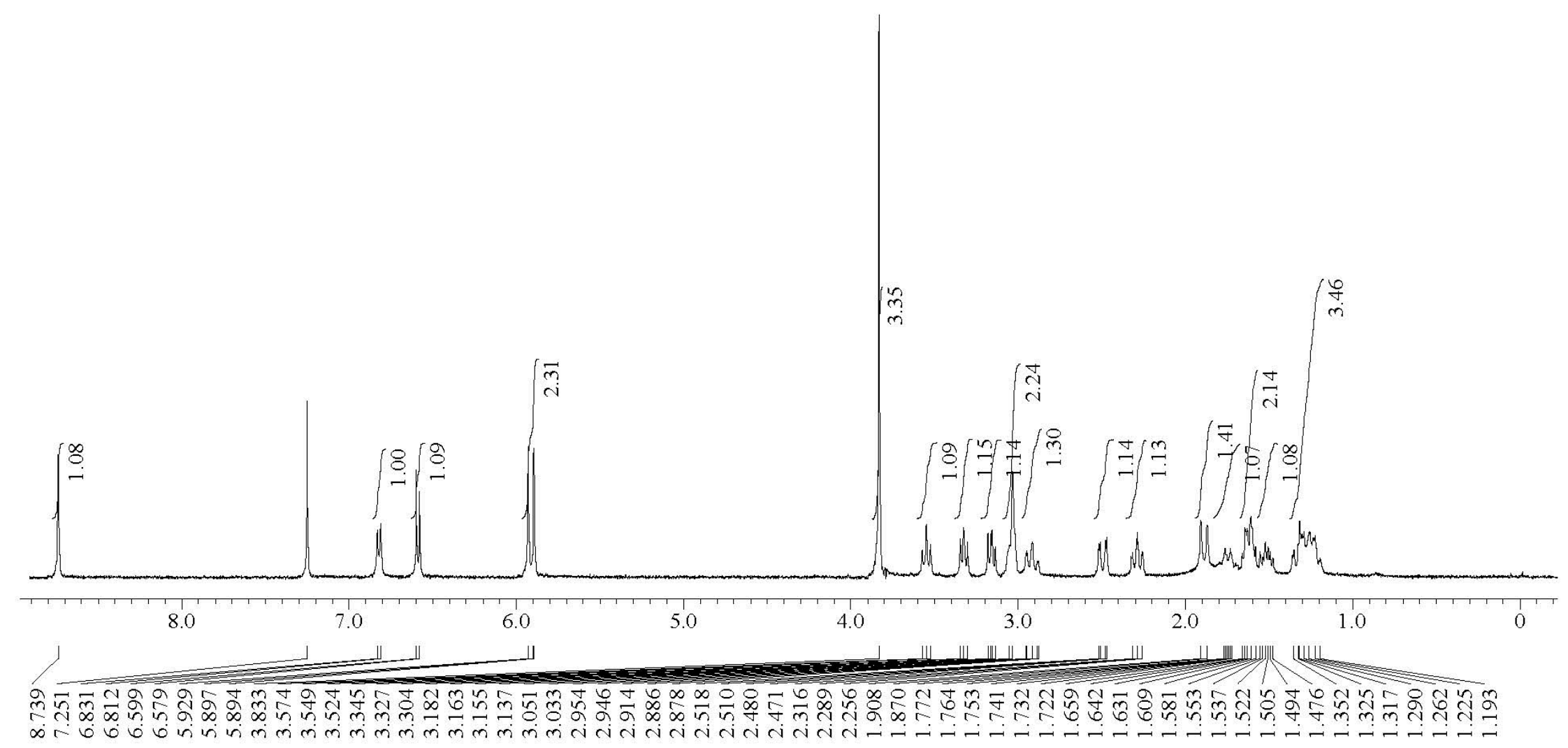

Figure S5. ${ }^{1} \mathrm{H}$ NMR Spectrum of Paucidactinine (3) $\left(\mathrm{CDCl}_{3}, 400 \mathrm{MHz}\right)$ 


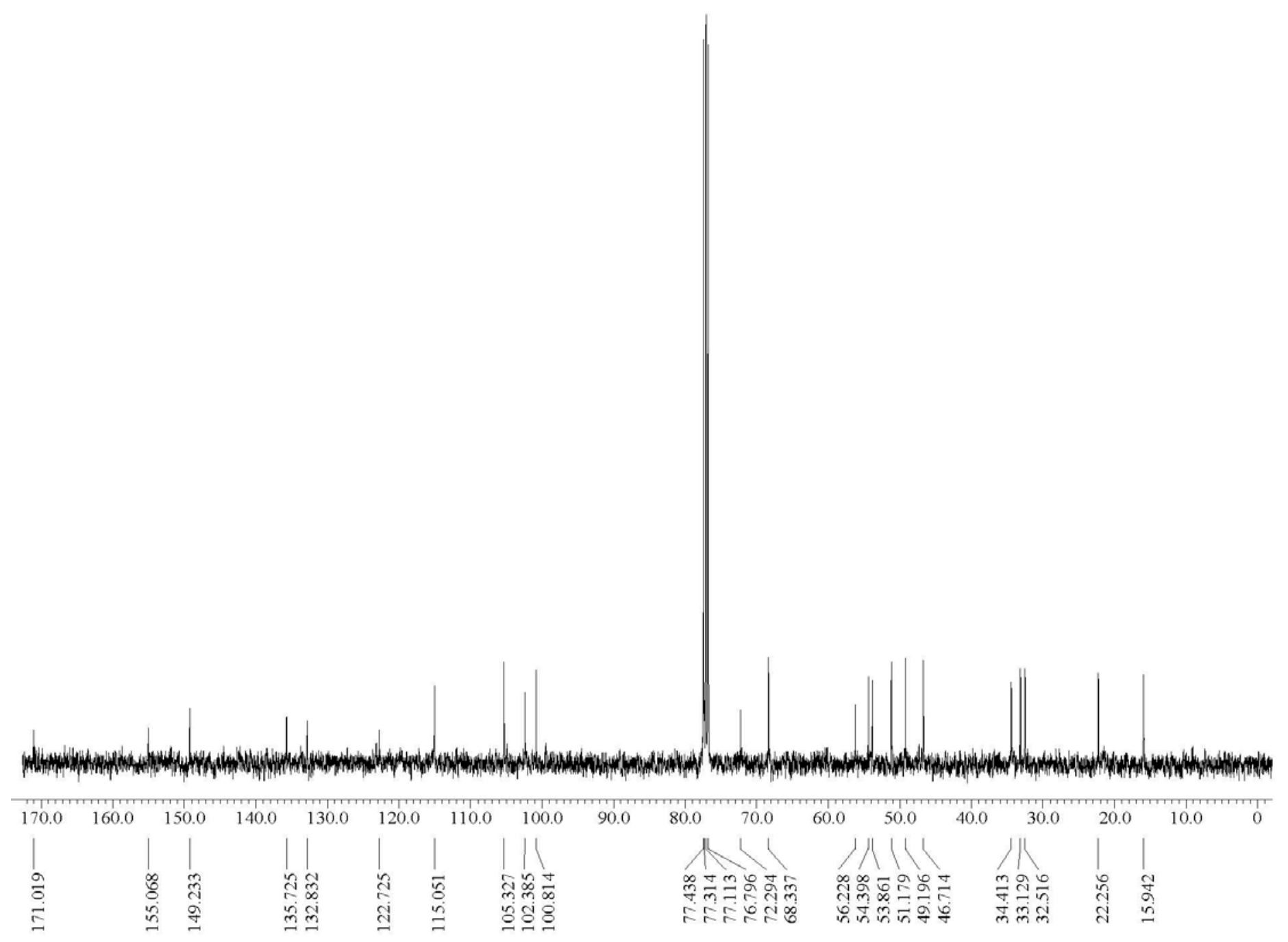

Figure S6. ${ }^{13} \mathrm{C}$ NMR Spectrum of Paucidactinine (3) $\left(\mathrm{CDCl}_{3}, 100 \mathrm{MHz}\right)$ 


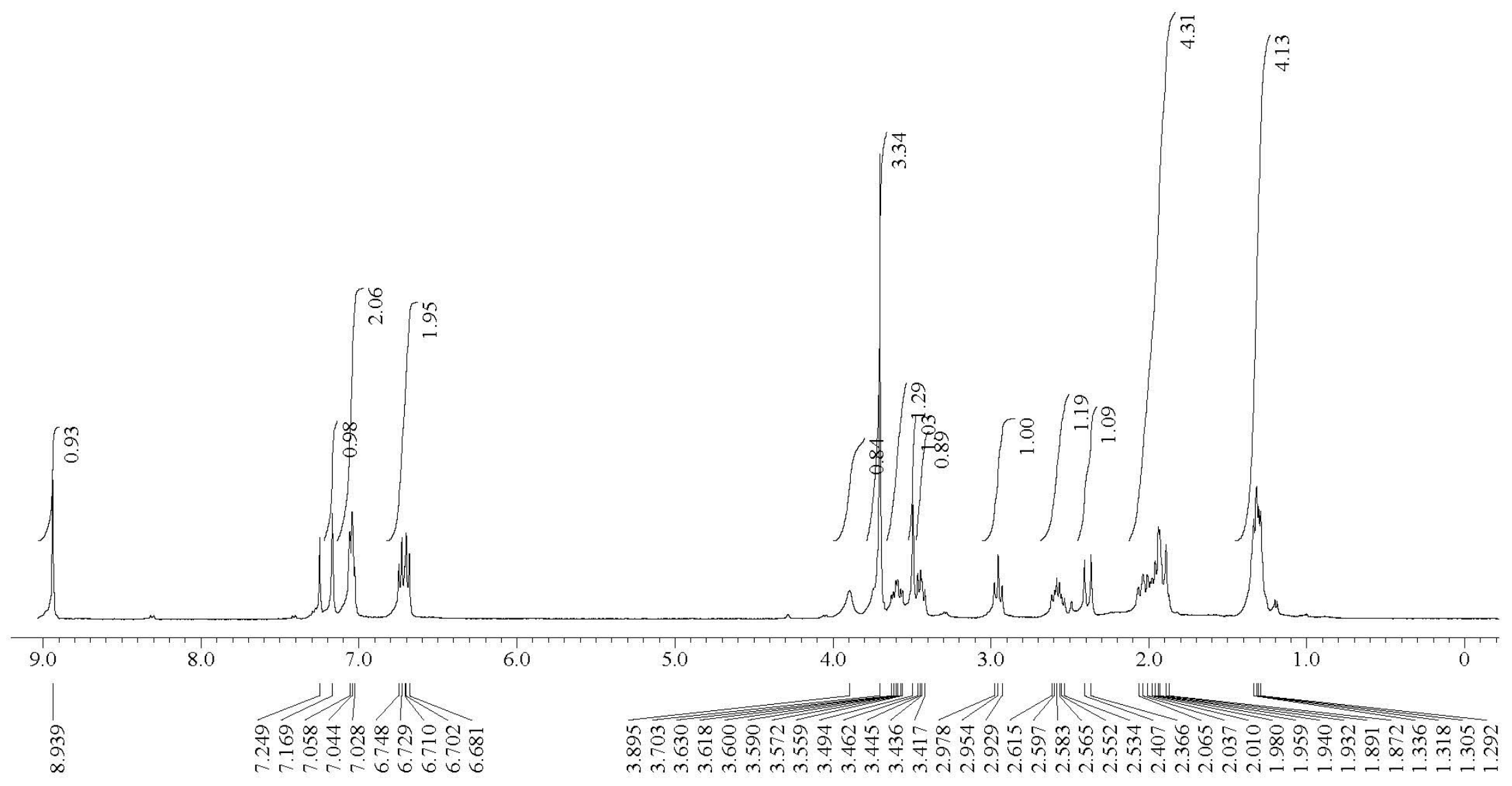

Figure S7. ${ }^{1} \mathrm{H}$ NMR Spectrum of Pauciduridine (4) $\left(\mathrm{CDCl}_{3}, 400 \mathrm{MHz}\right)$ 


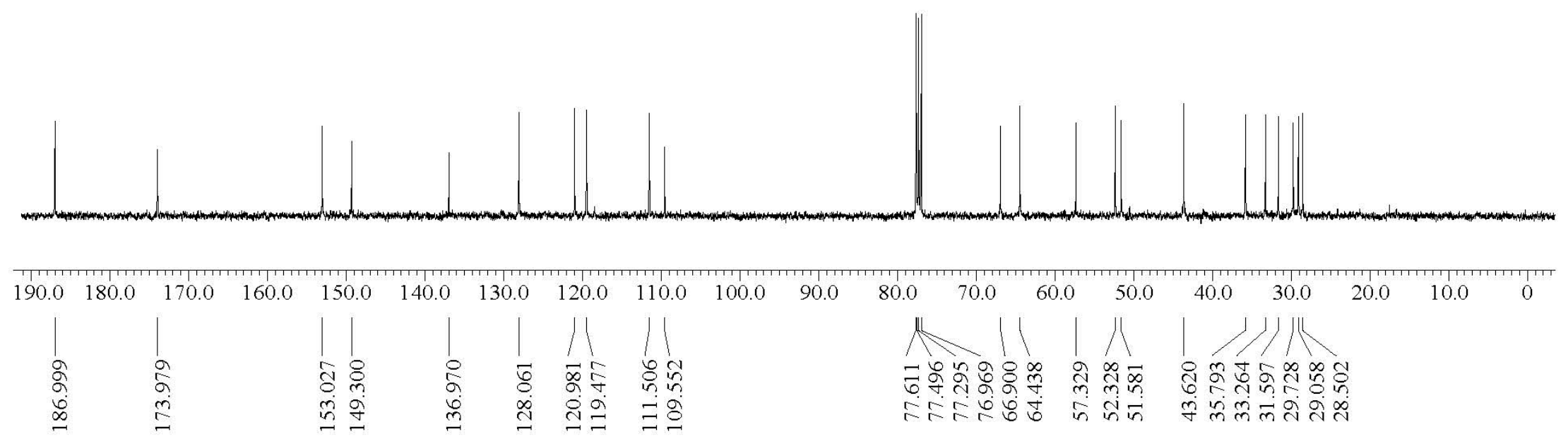

Figure S8. ${ }^{13} \mathrm{C}$ NMR Spectrum of Pauciduridine (4) $\left(\mathrm{CDCl}_{3}, 100 \mathrm{MHz}\right)$ 


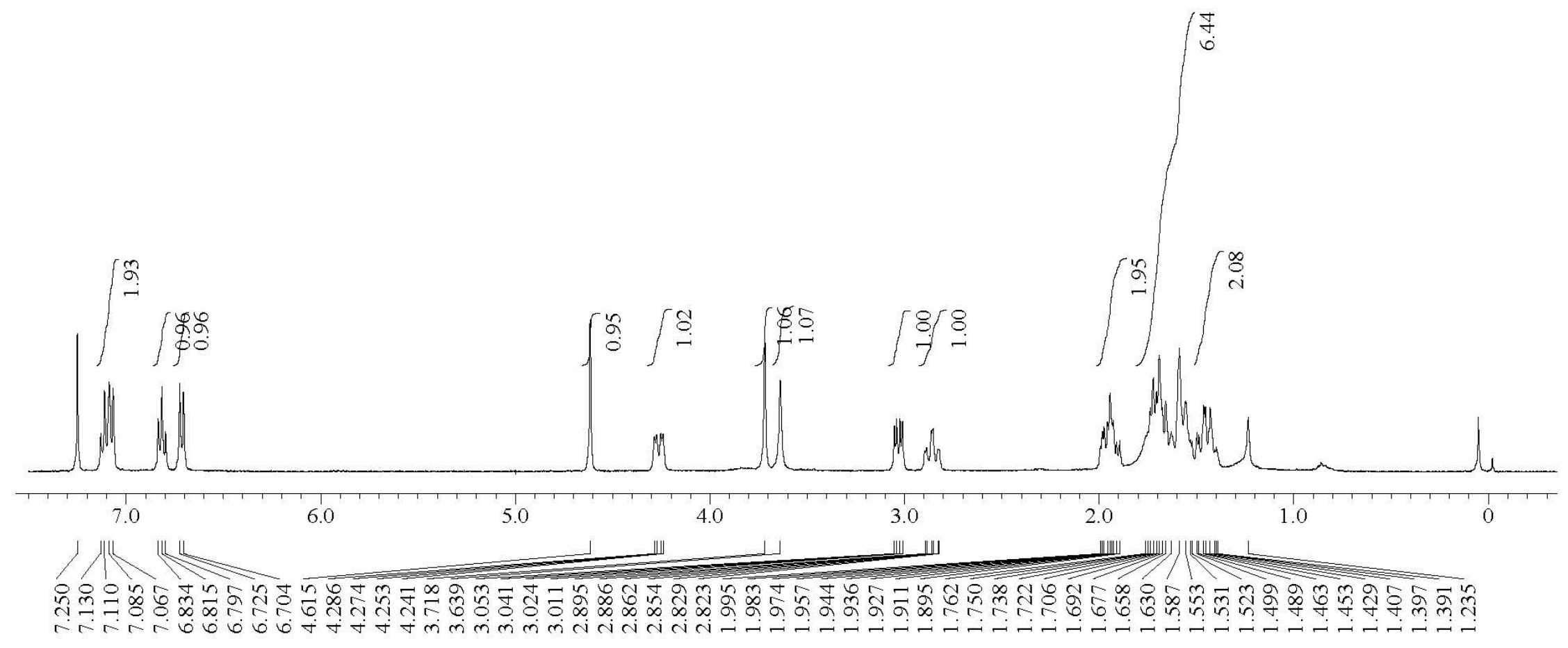

Figure S9. ${ }^{1} \mathrm{H}$ NMR Spectrum of Paucidactine D (5) $\left(\mathrm{CDCl}_{3}, 400 \mathrm{MHz}\right)$ 


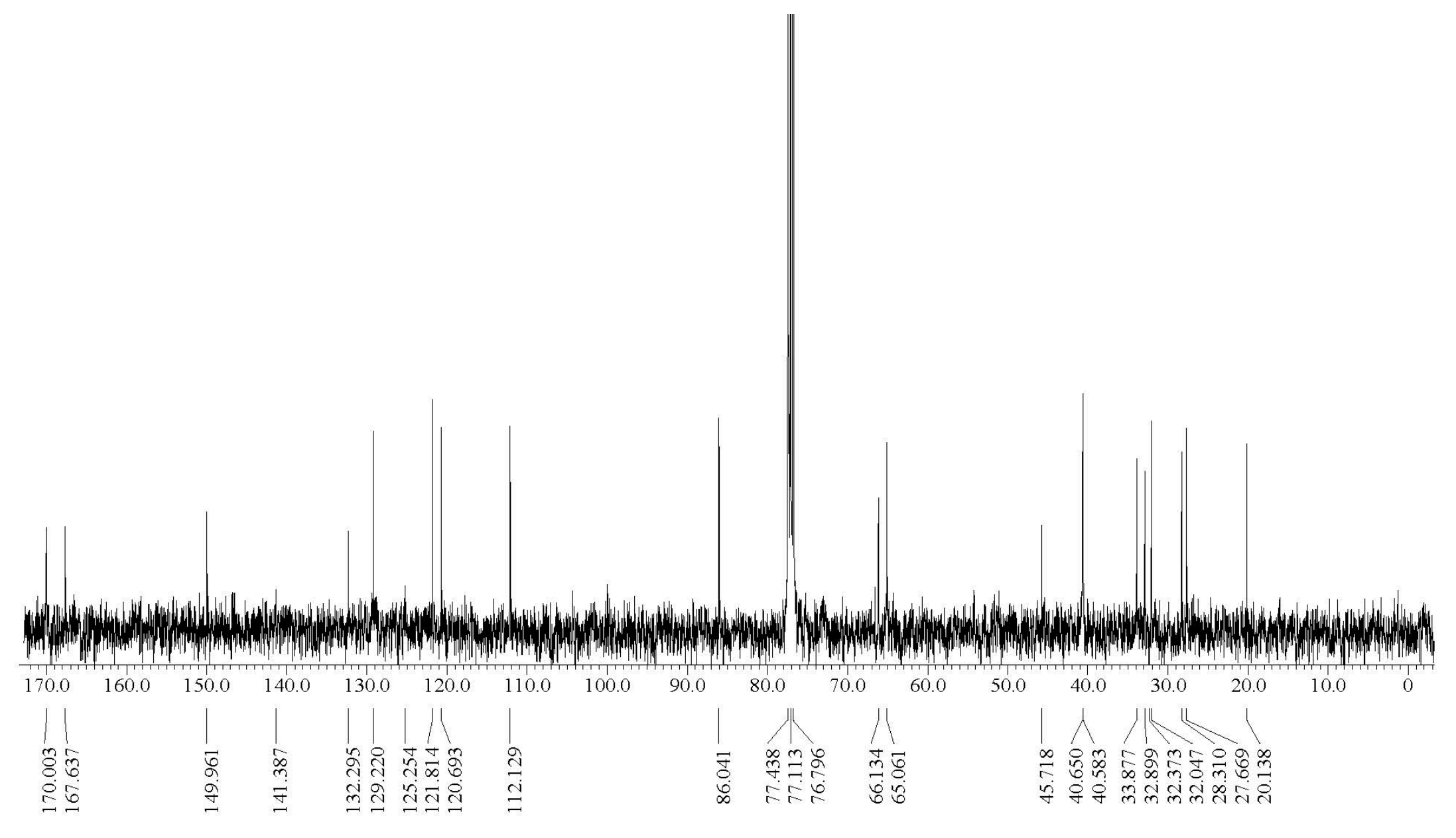

Figure S10. ${ }^{13} \mathrm{C}$ NMR Spectrum of Paucidactine D (5) $\left(\mathrm{CDCl}_{3}, 100 \mathrm{MHz}\right)$ 


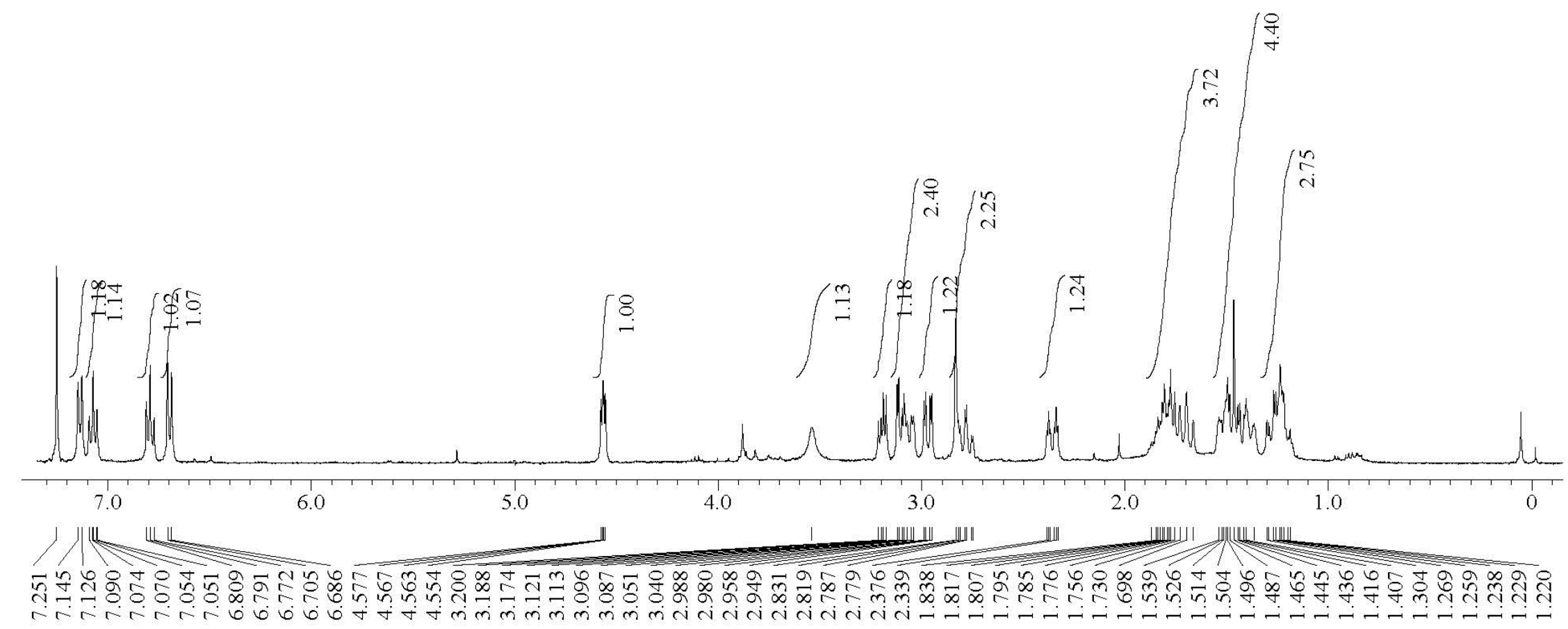

Figure S11. ${ }^{1} \mathrm{H}$ NMR Spectrum of Paucidactine E (6) $\left(\mathrm{CDCl}_{3}, 400 \mathrm{MHz}\right)$ 


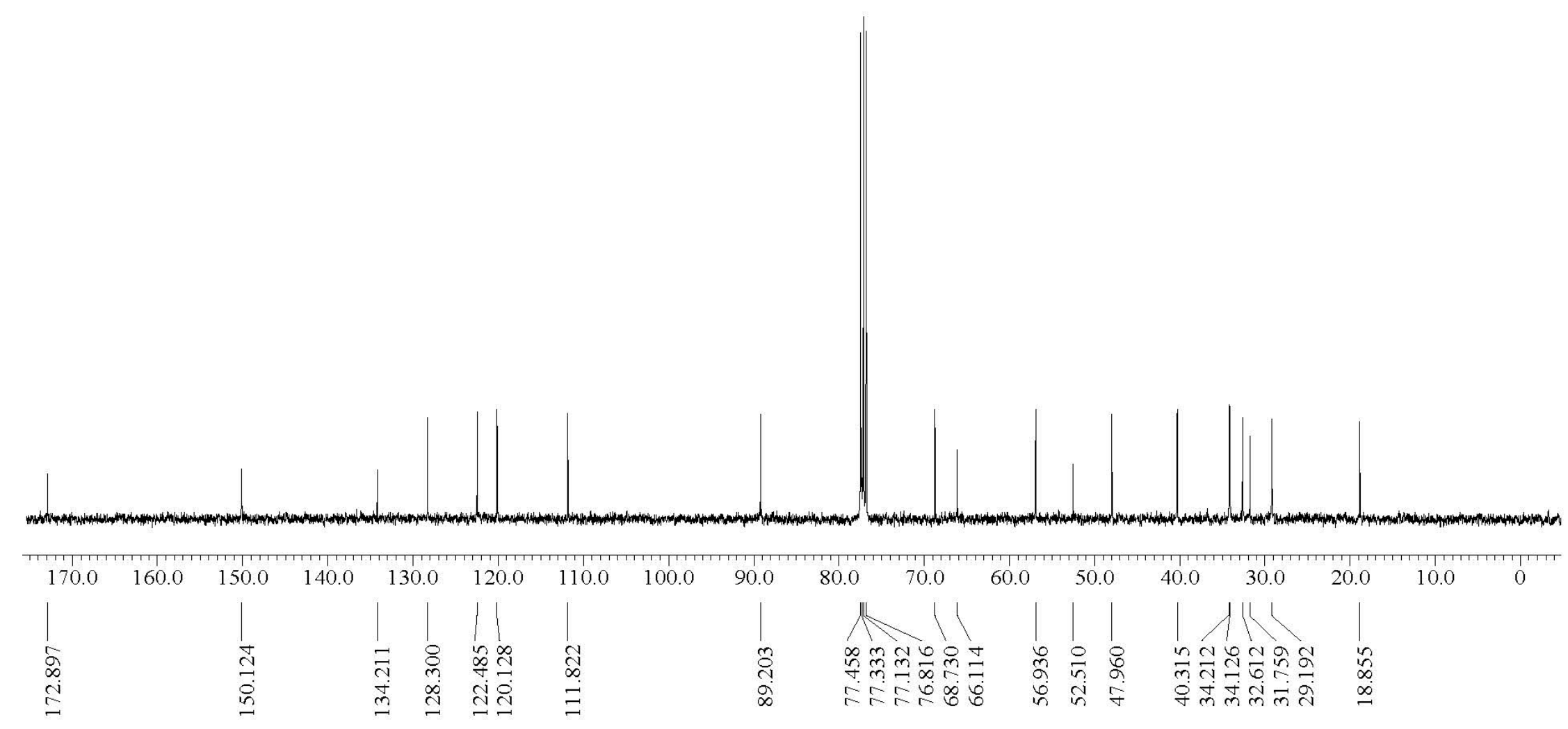

Figure S12. ${ }^{13} \mathrm{C}$ NMR Spectrum of Paucidactine E (6) $\left(\mathrm{CDCl}_{3}, 100 \mathrm{MHz}\right)$ 


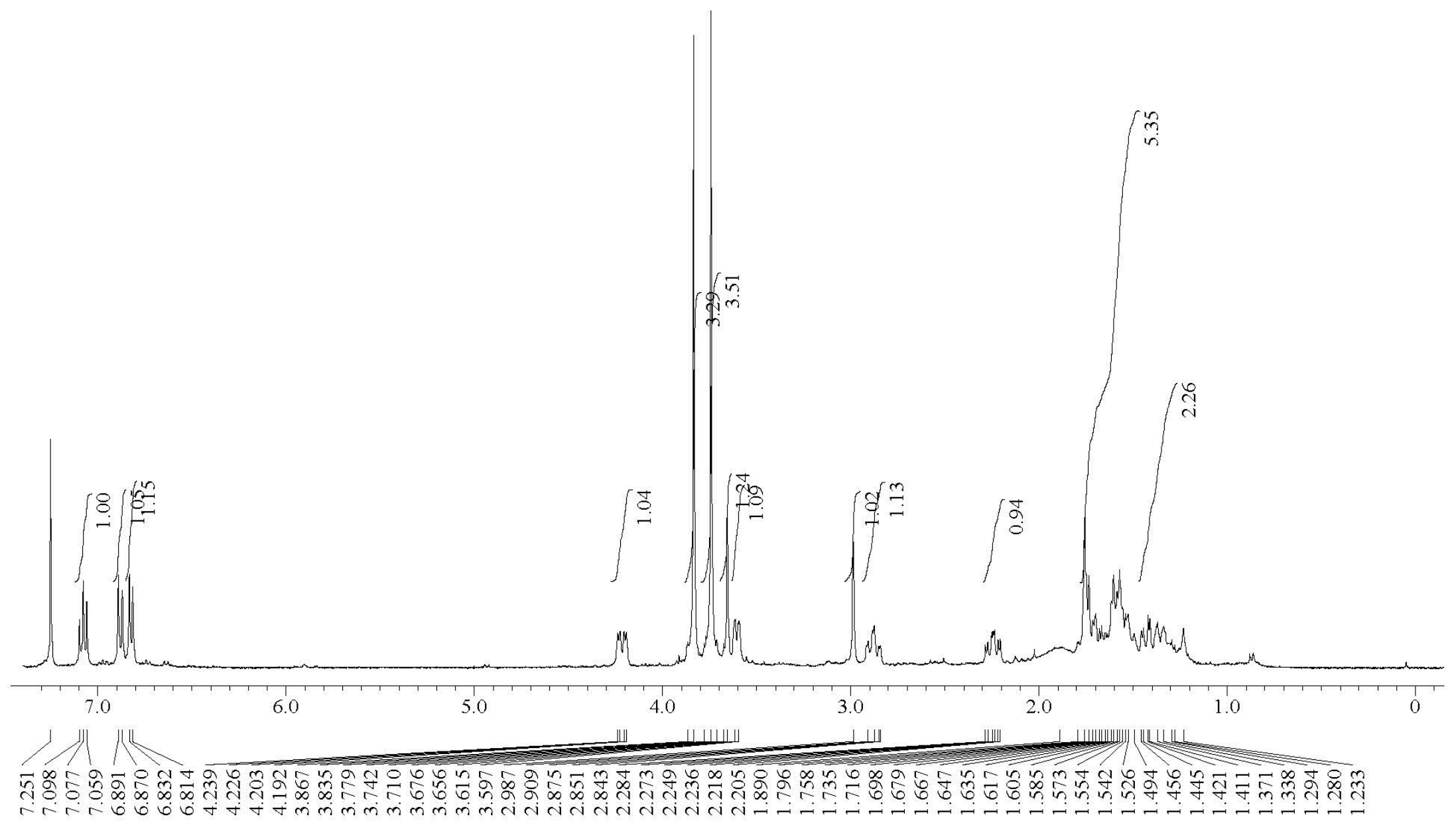

Figure S13. ${ }^{1} \mathrm{H}$ NMR Spectrum of Paucidisine (7) $\left(\mathrm{CDCl}_{3}, 400 \mathrm{MHz}\right)$ 


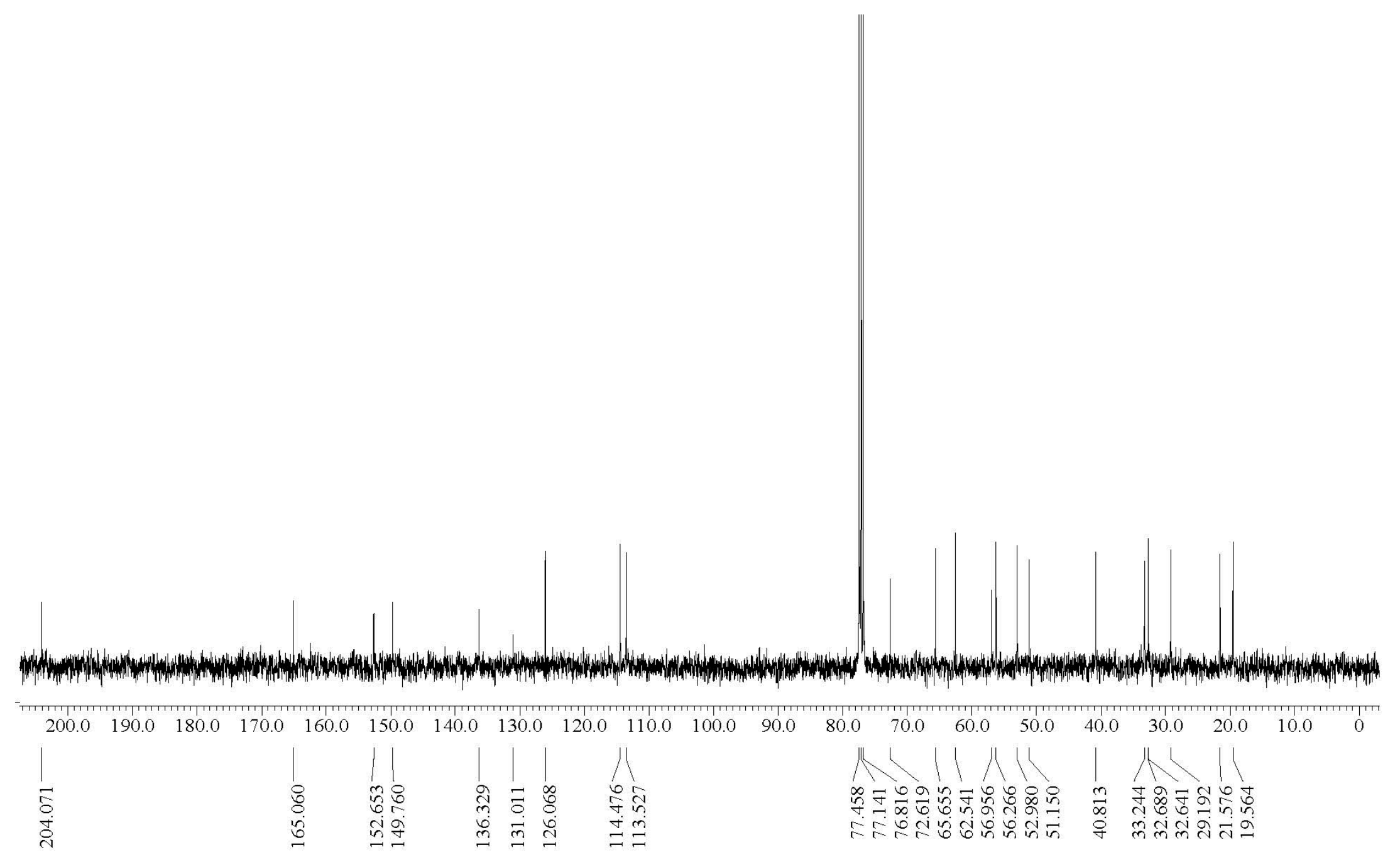

Figure S14. ${ }^{13} \mathrm{C}$ NMR Spectrum of Paucidisine (7) $\left(\mathrm{CDCl}_{3}, 100 \mathrm{MHz}\right)$ 


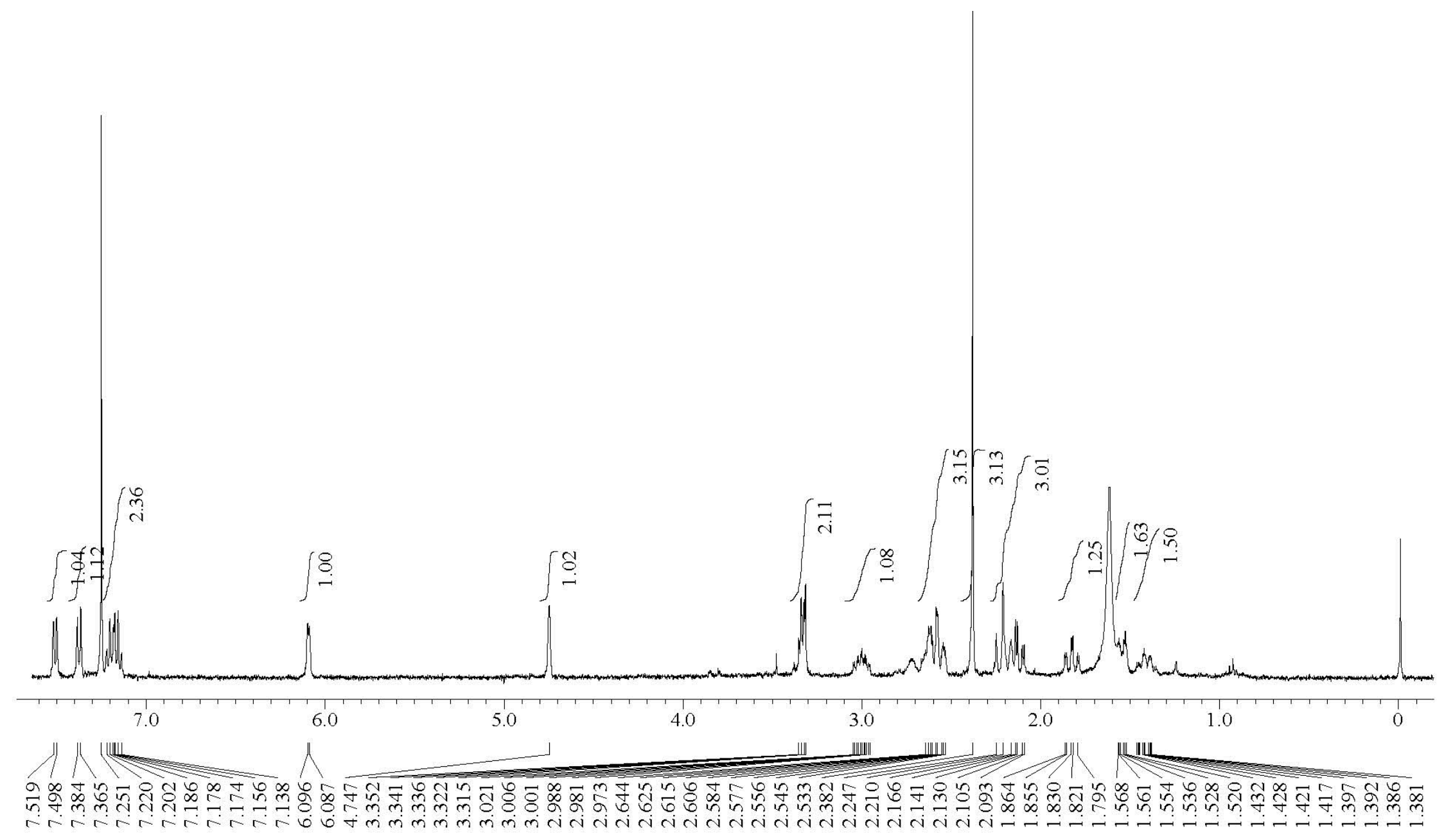

Figure S15. ${ }^{1} \mathrm{H}$ NMR Spectrum of (-)-19-oxoisoeburnamine (8) $\left(\mathrm{CDCl}_{3}, 400 \mathrm{MHz}\right)$ 


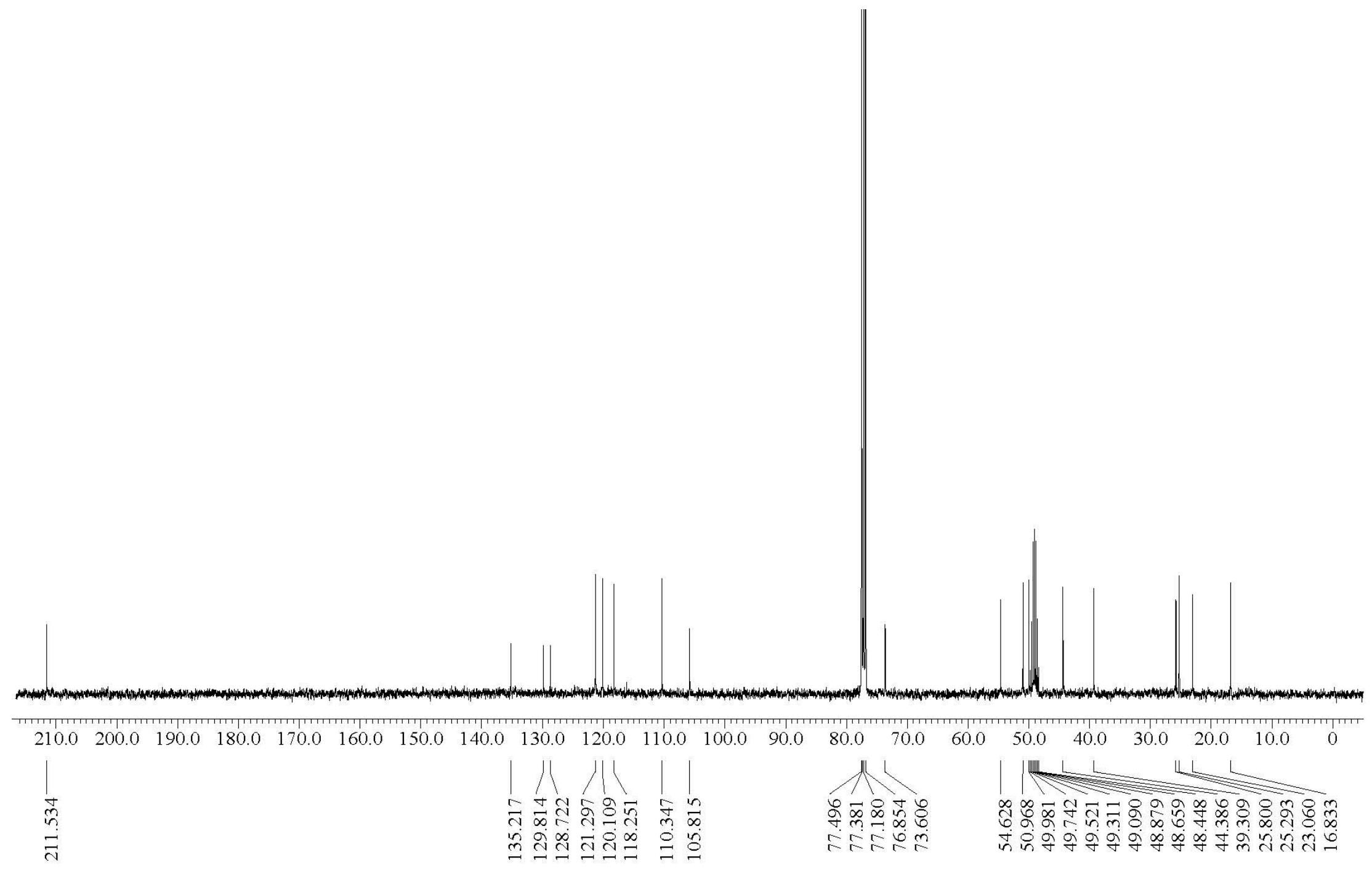

Figure S16. ${ }^{13} \mathrm{C}$ NMR Spectrum of (-)-19-oxoisoeburnamine (8) $\left(\mathrm{CDCl}_{3}, 100 \mathrm{MHz}\right)$ 


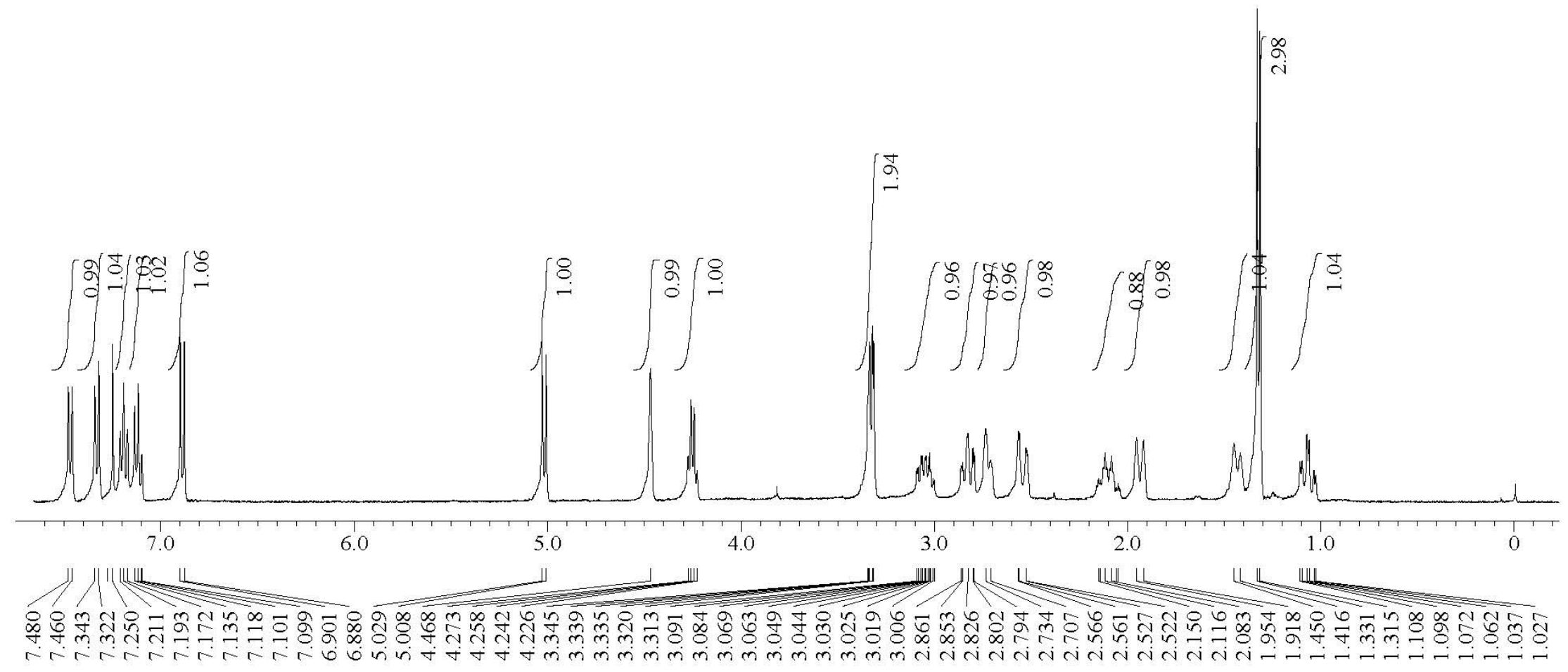

Figure S17. ${ }^{1} \mathrm{H}$ NMR Spectrum of (-)-19(R)-hydroxyeburnamenine (9) $\left(\mathrm{CDCl}_{3}, 400 \mathrm{MHz}\right)$ 


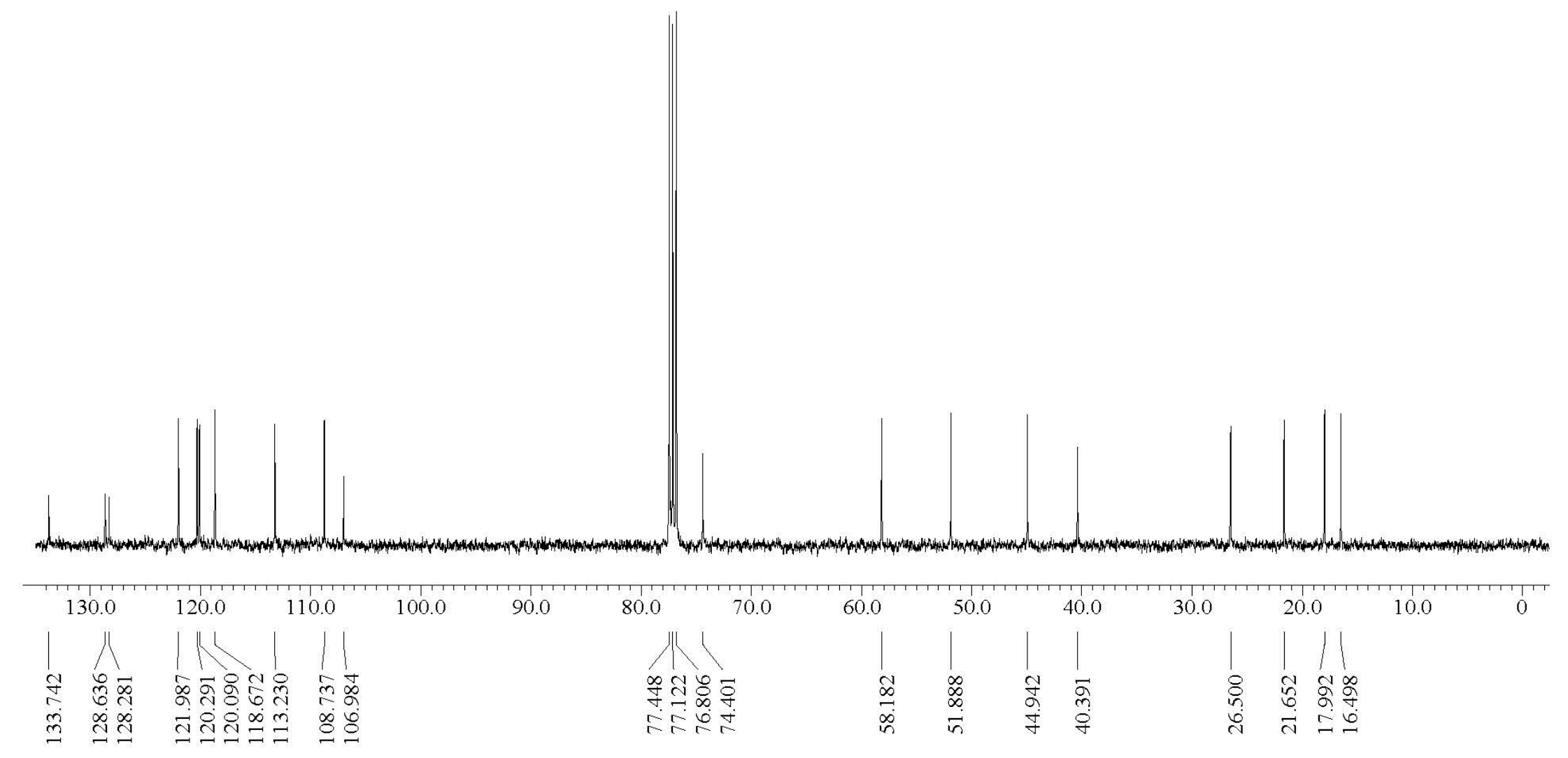

Figure S18. ${ }^{13} \mathrm{C}$ NMR Spectrum of (-)-19(R)-hydroxyeburnamenine (9) $\left(\mathrm{CDCl}_{3}, 100 \mathrm{MHz}\right)$ 


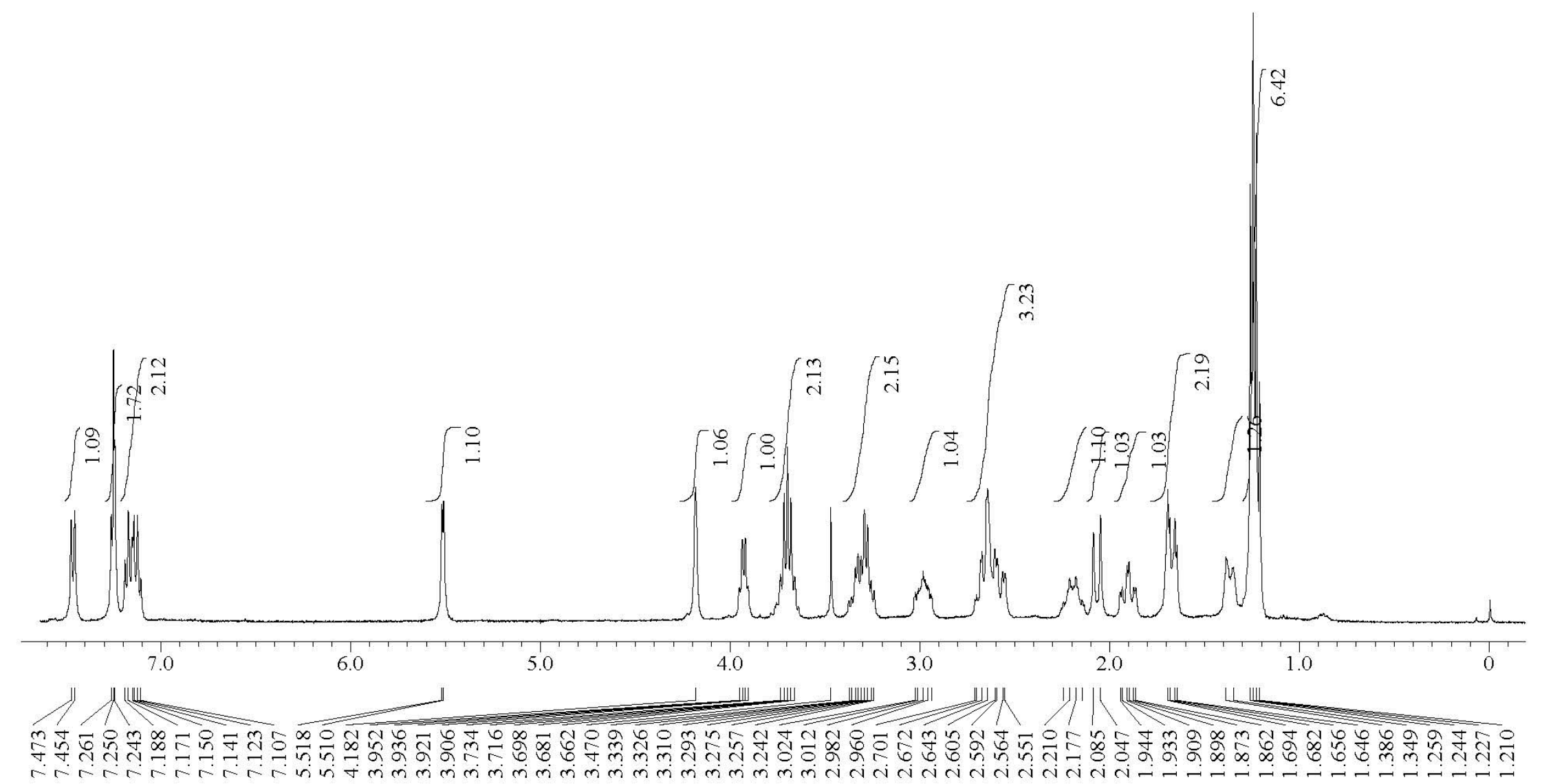

Figure S19. ${ }^{1} \mathrm{H}$ NMR Spectrum of (-)-19(R)-hydroxy-O-ethylisoeburnamine (10) $\left(\mathrm{CDCl}_{3}, 400 \mathrm{MHz}\right)$ 


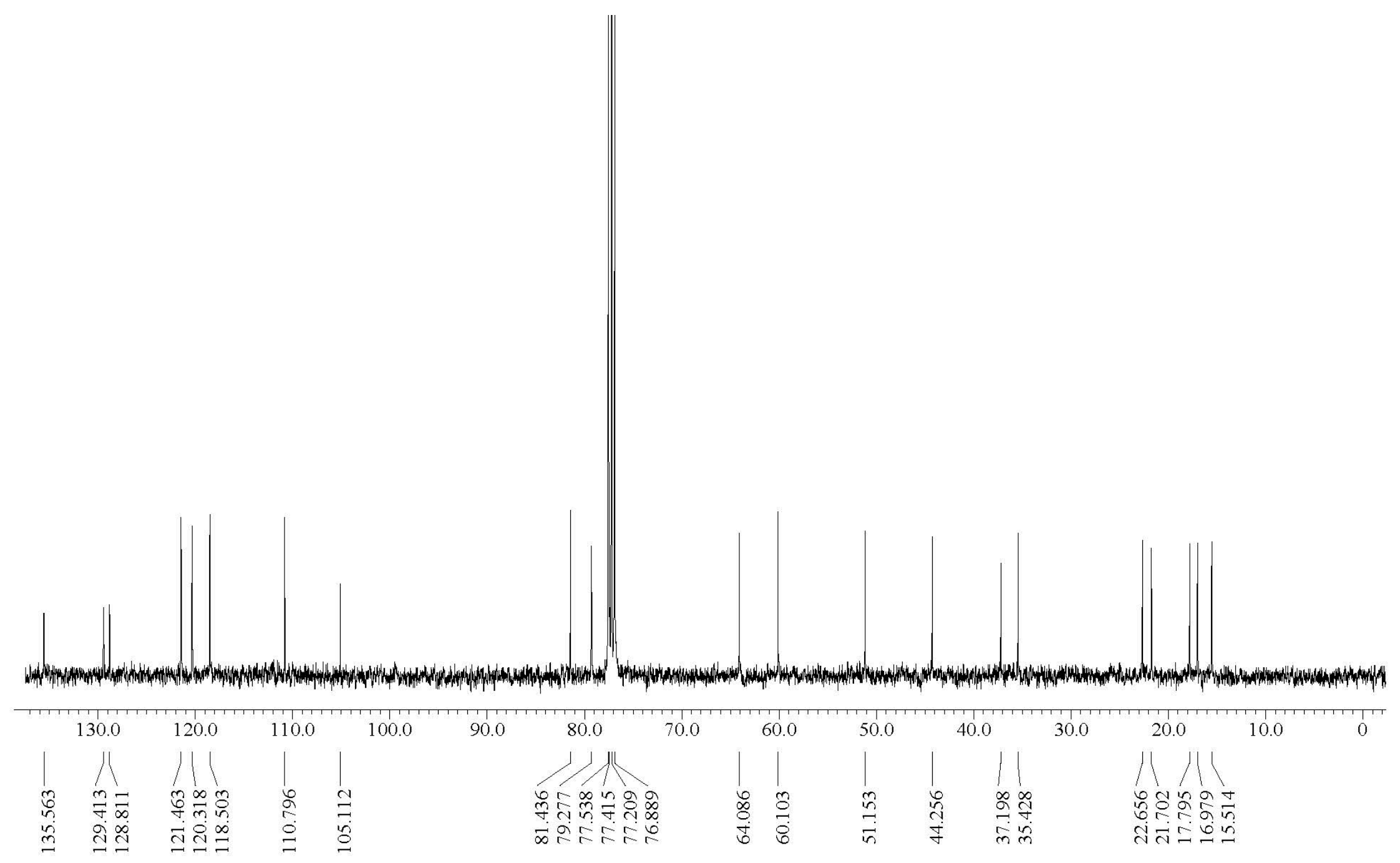

Figure S20. ${ }^{13} \mathrm{C}$ NMR Spectrum of (-)-19(R)-hydroxy-O-ethylisoeburnamine (10) $\left(\mathrm{CDCl}_{3}, 100 \mathrm{MHz}\right)$ 